\title{
openheart Device closure for patent foramen ovale following cryptogenic stroke: a survey of current practice in the UK
}

Katherine Von Klemperer, ${ }^{1}$ Aleksander Kempny, ${ }^{1}$ Christopher William Pavitt, ${ }^{2}$ John C Janssen, ${ }^{3}$ Anselm Uebing, ${ }^{4}$ Edward Nicol ${ }^{2}$

\begin{abstract}
- Additional material is published online only. To view, please visit the journal online (http://dx.doi.org/10.1136/ openhrt-2017-000636).
\end{abstract}

\section{To cite:}

Von Klemperer K, Kempny A, Pavitt CW, et al. Device closure for patent foramen ovale following cryptogenic stroke: a survey of current practice in the UK. Open Heart 2017;4:e000636. doi:10.1136/ openhrt-2017-000636

Abstract presented at CSI Conference 2016, Frankfurt

Received 1 April 2017 Revised 2 June 2017 Accepted 13 July 2017

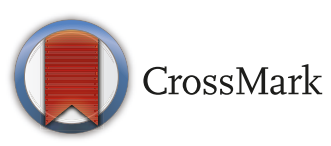

${ }^{1}$ Grown up Congenital Heart Disease Centre, Barts Heart Centre, London, UK

${ }^{2}$ Department of Cardiology, Royal Brompton Hospital, London, UK

${ }^{3}$ Department of Neurology, Chelsea and Westminster Hospital, London, UK

${ }^{4}$ Adult Congenital Heart Disease Centre, Royal Brompton Hospital, London, UK

Correspondence to Edward Nicol; cyprusdoc@ doctors.org.uk

\section{ABSTRACT}

Patent foramen ovale (PF0) closure for cryptogenic stroke remains controversial due to a lack of conclusive randomised controlled data. Many experts feel PFO closure is indicated in selected cases; however, national and international guideline recommendations differ. We surveyed the UK cardiologists, stroke physicians and neurologists, seeking to determine specialist interpretation of the evidence base, and to gain an insight into the current UK practice. The British Cardiac Society and British Society of Stroke physicians distributed our survey which was performed using an online platform. 120 physicians (70 stroke physicians, 23 neurologists, 27 cardiologists) completed the survey. Most ( $89 \%)$ felt PF0 closure should be considered in selected patients. Atrial fibrillation (86.6\%), significant carotid stenosis (86.6\%), diabetes (38.4\%) and hypertension $(36.6 \%)$ were considered exclusion criteria for cryptogenic stroke diagnosis. More stroke physicians than cardiologists considered an age cut-off when considering PF0 as the stroke aetiology $(70.4 \%$ vs $54.5 \% \mathrm{p}=0.04$ ). Anatomical features felt to support PF0 closure were aneurysmal septum (89.6\%), shunt size (73.6\%), prominent Eustachian valve (16\%). $60 \%$ discuss patients in multidisciplinary meetings prior to PFO closure, with more cardiologists than stroke physicians/neurologists favouring this approach $(76.9 \%$ vs $54.8 \% ; p=0.05)$. After PFO closure, patients receive Clopidogrel $(72.3 \%)$, aspirin $(50 \%)$ or anticoagulants (17\%). $63.2 \%$ continue therapy for a limited period after PF0 closure, while $34 \%$ prefer life-long therapy $(14.8 \%$ cardiologists vs $40.5 \%$ non-cardiologists; $p=0.02$ ). While experts support selective PFO closure in cryptogenic stroke, current practice remains variable with significant differences in perceptions of cardiologists and neurologists/stroke physicians.

\section{BACKGROUND}

The diagnosis and management of stroke related to patent foramen ovale (PFO) remains controversial. Observational studies and meta-analyses have suggested an association between PFO and cryptogenic stroke. However, the superiority of PFO closure over medical therapy for patients with cryptogenic stroke and PFO has proven particularly difficult to research due to multiple

\section{KEY MESSAGES}

What is already known about this subject? Evidence supporting the closure of patent foramen ovale (PFO) in patients with cryptogenic stroke to prevent recurrence has recently been published, but this is not reflected in all stroke, neurology and cardiology guidelines.

\section{What does this study add?}

This study shows a wide range of clinical practice in the UK with differences observed among stroke physicians, neurologists and cardiologists.

How might this impact on clinical practice? The results of this study, in combination with the 10year follow-up data from the RESPECT trial showing a benefit from PFO closure in patients with cryptogenic stroke, supports the need for standardised guidelines going forward to optimise patient selection and management.

confounding factors (such as concurrent traditional risk factors for stroke) and there is currently a lack of conclusive randomised controlled data to support device closure. ${ }^{1-3}$ Despite this, many experts continue to feel that PFO closure is indicated in selected cases (particularly in young patients, with high risk features such as atrial septal aneurysm (ASA) and/or large shunt size, and in those with no additional traditional risk factors). While the Federal Drug Administration has recently approved $\mathrm{PFO}$ device closure, and National and International guidelines propose diagnostic algorithms and patient selection criteria, ${ }^{4-6}$ these recommendations are still not uniform. Given the apparent inconsistency in advice and equivocal data to support PFO closure, we performed this survey of the UK cardiologists and stroke physicians/neurologists to determine specialist interpretation of the evidence base and to gain insight into the current UK practice. 


\section{Stroke Physicians}

Neurologists

\section{Cardiologists}

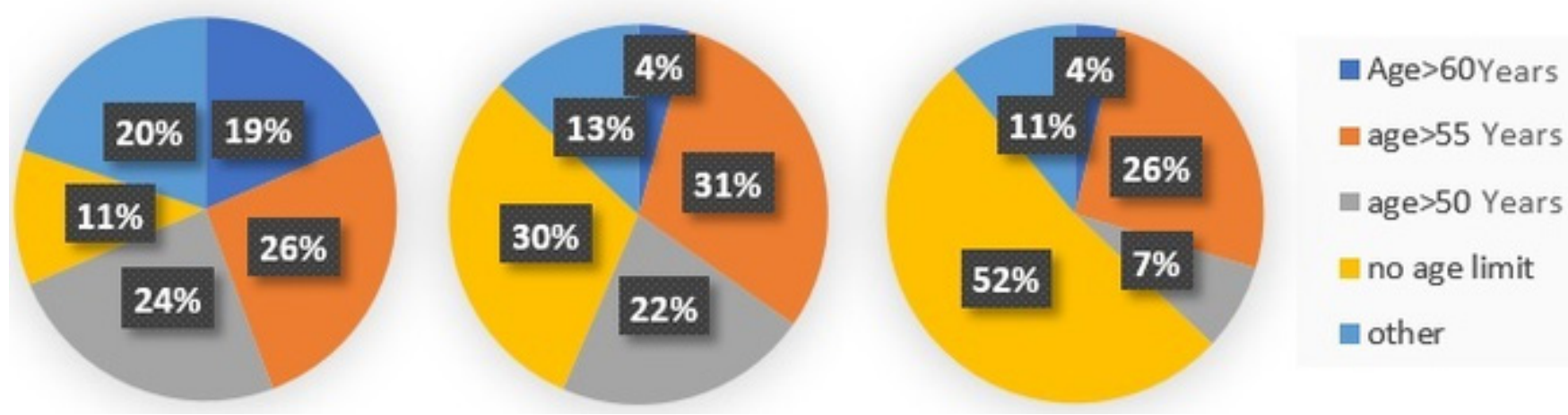

Figure 1 Reported age limit above which responders would not consider patent foramen ovale (PFO) closure. There is significantly higher proportion of cardiologists compared with stroke physicians reporting no age limit for PFO closure $(p=0.04)$.

\section{METHODS}

In this cross-sectional study carried out between 20 December 2014 and 22 February 2015, an online survey was distributed (www.surveymonkey.com) (see online supplementary 1) via the British Cardiac Society, the British Society of Stroke Physicians and the Association of British Neurologists. The survey was designed by the authors and designed to capture data on the respondent's clinical experience, consider indications and criteria for PFO closure, exclusion criteria, imaging, multidisciplinary team management and anticoagulation therapy after device insertion. After a 3-month reporting period, responses were collated and analysed. Although the survey was not specifically powered to determine differences between responders, where differences in practice were apparent a $\mathrm{X}^{2}$ test was used to assess the differences in responses. A p value $<0.05$ was considered to be statistically significant.

\section{RESULTS}

\section{Study population}

A total of 120 individuals responded, the majority of whom were stroke physicians. Not all respondents answered all questions and therefore data are presented as a proportion of the total number of respondents for each individual question.

\section{PFO closure practice}

The majority of respondents (108/120 (89\%)) felt there was a role for PFO closure in selected patients with cryptogenic stroke. More neurologists and stroke physicians than cardiologists felt that there is currently insufficient evidence to support PFO closure after stroke $(13 / 90(14.4 \%)$ vs $1 / 27(3.7 \%) ; p=0.11)$. There was also a non-significant trend towards younger consultants ( $<5$ years in consultant position) believing the current evidence does not support PFO closure $(6 / 13$ (46\%) vs $22 / 107(20.6 \%) ; \mathrm{p}=0.09)$. The majority $(78 / 112(69.6 \%))$ of responders considered ischaemic stroke (cerebrovascular accident (CVA)) as well as transient ischaemic attack (TIA) as cerebrovascular events warranting further assessment for PFO. Approximately one-third (33/112 $(29.5 \%)$ ) considered CVA, but not TIA, as a valid reason for further evaluation. There was a trend for cardiologists to consider PFO assessment for both stroke and TIA in comparison to stroke physicians $(21 / 26(81 \%)$ vs $41 / 63$ $(65.1 \%) ; \mathrm{p}=0.06)$, but not neurologists $(16 / 23(70.0 \%)$; $\mathrm{p}=0.31)$.

Seventy-one out of one hundred ten (64.5\%) responders would consider PFO closure after one cerebrovascular event, $23 / 110(20.9 \%)$ two events and the remainder $(16 / 110(14.5 \%))$ only after three or more events. Overall, cardiologists had a significantly lower threshold for considering device closure than non-cardiologists after a single cerebral event $(50 / 84(60.0 \%)$ vs $21 / 26(81.0 \%) ; \mathrm{p}=0.041)$, which was driven by a significant difference between cardiologists and neurologists $(21 / 26(81.0 \%)$ vs $1 / 22(45.5 \%) ; p \leq 0.01)$.

\section{Evaluation for PFO closure}

While the majority $(92 / 114(80.7 \%))$ of responders required evidence of stroke on CT or MRI prior to considering PFO closure, cardiologists were less likely than neurologists to require such evidence (19/26 (73.1\%) vs $19 / 23(82.6 \%) ; \mathrm{p}=0.04)$. A quarter of responders $(29 / 113,(25.7 \%))$ did not consider an age cut-off for $\mathrm{PFO}$ closure, while cardiologists were statistically more likely to consider patients of any age for $\mathrm{PFO}$ closure $(14 / 27$ (52\%) vs $15 / 86(17 \%) ; \mathrm{p}<0.001)$. A greater proportion of stroke physicians considered an age cut-off when considering PFO as the aetiology of stroke than cardiologists $(38 / 54(70.4 \%)$ vs $20 / 37$ (54.5\%); $\mathrm{p}=0.04$ ) (figure 1). Significant carotid stenosis or atrial fibrillation $(87.5 \%$ and $86.6 \%$ responders, respectively) were considered exclusion criterion for a diagnosis of cryptogenic stroke. The presence of hypercholesterolaemia, diabetes mellitus, arterial hypertension, calcific aortic valve disease or other comorbidities were considered important exclusion criteria by less than half of the responders (see figure 2). There were no observed 


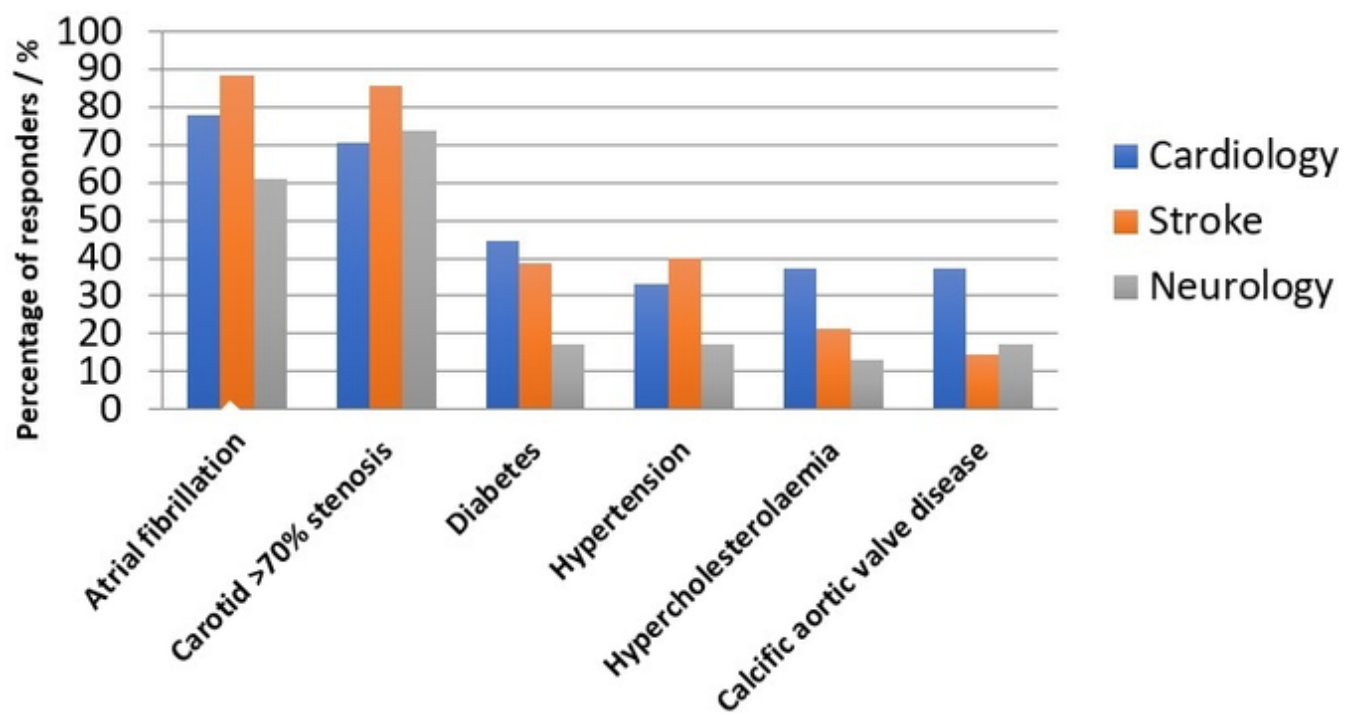

Figure 2 Comorbidities excluding from further evaluation for patent foramen ovale closure.

differences in practice between cardiologists, neurologists and stroke physicians.

Almost all responders $(111 / 114(97.4 \%))$ felt the diagnosis of PFO should be confirmed with a transthoracic echocardiogram (TTE) 'bubble study' with several features deemed to increase the likelihood that a PFO was implicated in the aetiology a cryptogenic stroke; these included the presence of an aneurysmal septum and increased shunt size on TTE (see figure 3). Nearly three-quarters $(84 / 113,74.3 \%)$ of responders were more likely to proceed to PFO device closure based on the presence of these anatomical features, while (21/113 $(18.6 \%))$ did not take these features into account in decision-making. Cardiologists and stroke physicians differed on the significance of certain echocardiographic features (eg, aneurysmal septum, shunt size) when deciding whether a PFO was implicated in the neurological event $(\mathrm{p}=0.04)$. Almost all $(108 / 111 \quad(97.3 \%))$ physicians responding included a carotid artery scan and Holter ECG monitoring (99/111 (89.2\%)) as part of their workup for cryptogenic stroke.

\section{Management of PFO closure}

Sixty-six out of one hundred ten $(60 \%)$ of responders felt it necessary to formally discuss patients in a multidisciplinary team meeting (MDT) prior to PFO device closure

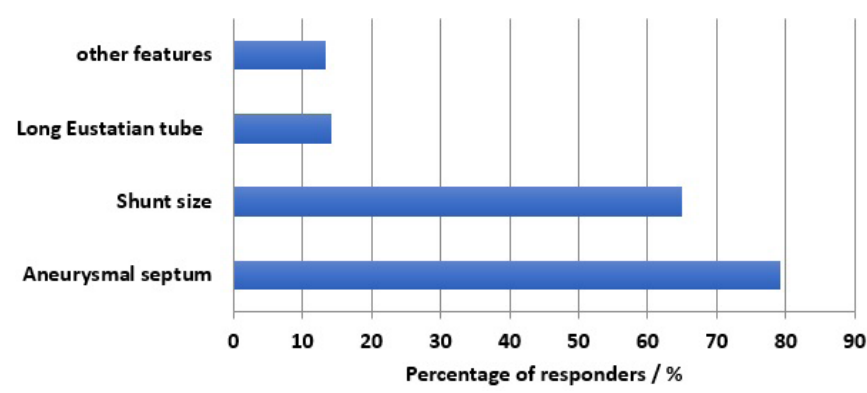

Figure 3 Anatomical features associated with increased probability of cryptogenic stroke. with more cardiologists than either stroke physicians or neurologists favouring this approach (20/26 (76.9\%) vs $46 / 84(54.8 \%) ; \mathrm{p}=0.045)$. Additionally, more stroke physicians than neurologists also felt an MDT necessary for decision-making $(35 / 60(58.3 \%)$ vs $1 / 12(8.3 \%)$; $\mathrm{p}=0.03$ ). Where undertaken, MDT composition usually consisted of an interventional cardiologist (80.6\%), neurologist $(65.3 \%)$, imaging cardiologist/radiologist $(48.5 \%)$ and congenital heart disease cardiologist $(41.7 \%)$; only $8.3 \%$ always had a haematologist present.

Following PFO device closure, most respondents favoured either Clopidogrel $(81 / 112(72.3 \%))$, acetylsalicylic acid (ASA) $(56 / 112(50 \%))$ or warfarin therapy $(15 / 112(13.4 \%))$ (figure 4$)$. Two-thirds of responders $(67 / 106(63.2 \%))$ use antithrombotic agents or anticoagulation for a limited period of time after PFO closure, while (36/106 (34\%)) advocate life-long therapy. Overall, significantly fewer cardiologists believe life-long therapy is required, as compared with non-cardiologists $(4 / 27$ $(14.8 \%)$ vs $32 / 79(40.5 \%) ; p=0.021)$.

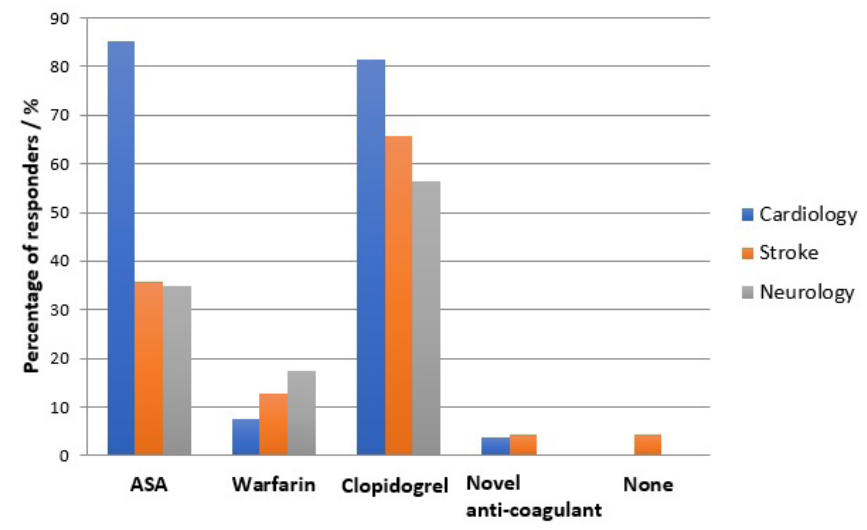

Figure 4 Medical therapy postpatent foramen ovale device closure. ASA, acetylsalicylic acid; NOAC, novel anticoagulant. 


\section{DISCUSSION}

Until 2012, and in accordance with guidance from the National Institute of Healthcare Excellence (NICE) (NICE guidance IPG 109 Secondary Prevention of recurrent paradoxical emboli), patients who suffered a presumed paradoxical, CT/MRI confirmed cerebral vascular event, who were found to have a PFO with inducible right to left shunting, were eligible for PFO closure device. This led to widespread adoption of PFO closure for cryptogenic stroke, with a $48.4 \%$ increase in the number of PFO closure procedures in the UK over a 4-year period. ${ }^{7}$ However, because of insufficient supporting evidence from prospective trials and meta-analyses, and concerns regarding cost-effectiveness, NHS England withdrew routine commissioning for PFO device closure in 2014. ${ }^{3-10}$ Until December 2016, PFO device closure was still permitted in England, however only through the NHS England Commissioning through Evaluation programme. This programme ceased in mid-December 2016 and further advice and guidance is awaited from NHS England as the specialist care commissioner for this service.

This survey clearly demonstrates an inconsistent approach to the selection of patients considered for PFO closure following a cerebrovascular event from a presumed cryptogenic source. These inconsistencies include approach to imaging and MDT discussion prior to device closure and subsequent antiplatelet/anticoagulation management, with significant differences in practice between different specialists.

While the majority of respondents still feel that there remains a place for PFO closure for cryptogenic stroke, despite inconclusive evidence from recent major trials, there were significant differences in the way cryptogenic stroke was defined and which comorbidities should exclude PFO device closure. The recent Randomized Evaluation of Recurrent Stroke Comparing PFO Closure to Established Current Standard of Care Treatment (RESPECT) 10-year outcome data, published after this survey, highlight the importance of careful patient selection. In their subanalysis of patients with recurrent cryptogenic strokes, the investigators showed that the PFO occluder remained superior to medical management alone (HR 0.38, p=0.007). This effect was magnified further when the population was narrowed to only include patients aged under 60 years. ${ }^{11}$

In our survey, more cardiologists believed PFO device closure is indicated after both stroke and TIA versus stroke alone and considered single versus recurrent events as a positive indication for device closure, compared with neurologists and stroke physicians. Additionally, a significantly lower percentage of cardiologists than neurologists/stroke physicians believe an age cut-off is appropriate when implicating PFO in cryptogenic stroke. This possibly suggests cardiologists have a less conservative approach to their selection of patients with cerebral events for PFO closure. Interestingly, while most respondents felt atrial fibrillation and significant carotid stenosis should exclude individuals from PFO device closure, other significant risk factors for cerebrovascular events such as diabetes, hypertension, hypercholesterolaemia and calcific aortic stenosis were felt relevant by barely half of the respondents.

The Risk of Paradoxical Embolism (ROPE)study and RESPECT trials among others have associated certain anatomical features of PFO with cryptogenic stroke. ${ }^{12}$ The majority of our respondents agreed that an aneurysmal septum or large shunt were more likely to implicate the PFO in stroke; however, not all respondents took these features into account in their decision to undertake device closure. Cardiologists were more inclined than neurologists/stroke physicians towards an inclusive MDT process prior to intervention.

Following device insertion, there was considerable variation in the approach to antiplatelet therapy and anticoagulation. Clopidogrel, as a single agent was, by far, the preferred option following device closure; however, cardiologists were significantly more likely to use aspirin than stroke physicians/neurologists, with a slight preference for warfarin by stroke physicians and neurologists. Interestingly, RESPECT has just shown an increased incidence of DVT and PE in the group of patients randomised to device closure compared with those on medical therapy (a greater proportion of whom were on anticoagulants) $(\mathrm{p}<0.006){ }^{11}$

Two-thirds of all respondents recommended antiplatelet therapy for a limited time after PFO closure, while a third believed it should be life-long, again with a statistically significant difference in preference between specialist groups. There was also a clear lack of consensus as to when, and how often, patients should be seen post-PFO closure.

\section{Limitations}

This is a small and simple survey designed to capture specialist opinions on perception of the role of $\mathrm{PFO}$ device closure in cryptogenic stroke. While all respondents were asked about the number of patients they see per year with cryptogenic stroke where PFO device i considered, there is no indication as to the level of involvement of respondents in the decision-making process for device closure. Additionally, it cannot be determined to exactly how many consultants this survey was distributed to and therefore may be more biased towards those who have a vested interest in this procedure being undertaken. Furthermore, there is no correlative data as to the number of devices inserted in the institutions of the individual respondents. Due to these limitations, care should be exercised in the determination as to the level of evidence that can be attached to this dataset and its generalisability. However, it does give an insight into the apparent perceptions and practice of $\mathrm{PFO}$ device closure in cryptogenic stroke in the UK. 


\section{CONCLUSIONS}

This study suggests that while most experts do feel there is a place for PFO closure for cryptogenic stroke, there remain marked differences in their approach to investigating and managing these patients and in particular significant difference between the approach taken by cardiologists, neurologists and stroke physicians. Most specialists are seeing small numbers of these patients on a yearly basis. The results of this study, in combination with the 10-year follow-up data from the RESPECT trial showing a benefit from PFO closure in patients with cryptogenic stroke, supports the need for standardised guidelines going forward to optimise patient selection and management.

Contributors KVK, AK, JJ, AU and ED were responsible for study conception and design of the questionnaire. $\mathrm{CP}$ and $\mathrm{AK}$ were responsible for data analysis and interpretation. KVK and CP were responsible for drafting of the article. All authors contributed to revising the intellectual content of the article and approved it for final publication.

Funding Funded by research grant from NHS England Armed forces Clinical Reference Group

Competing interests None declared.

Provenance and peer review Not commissioned; externally peer reviewed.

Open Access This is an Open Access article distributed in accordance with the Creative Commons Attribution Non Commercial (CC BY-NC 4.0) license, which permits others to distribute, remix, adapt, build upon this work non-commercially, and license their derivative works on different terms, provided the original work is properly cited and the use is non-commercial. See: http://creativecommons.org/ licenses/by-nc/4.0/

(c) Article author(s) (or their employer(s) unless otherwise stated in the text of the article) 2017. All rights reserved. No commercial use is permitted unless otherwise expressly granted.

\section{REFERENCES}

1. Agarwal S, Bajaj NS, Kumbhani DJ, et al. Meta-analysis of transcatheter closure versus medical therapy for patent foramen ovale in prevention of recurrent neurological events after presumed paradoxical embolism. JACC Cardiovasc Interv 2012;5:777-89.

2. Li J, Liu J, Liu M, et al. Closure versus medical therapy for preventing recurrent stroke in patients with patent foramen ovale and a history of cryptogenic stroke or transient ischemic attack. Cochrane Database Syst Rev 2015;9:CD009938.

3. Furlan AJ, Reisman M, Massaro J, et al. Closure or medical therapy for cryptogenic stroke with patent foramen ovale. N Engl J Med 2012;366:991-9.

4. European Stroke Organisation (ESO) Executive CommitteeESO Writing Committee. Guidelines for management of ischaemic stroke and transient ischaemic attack 2008. Cerebrovasc Dis 2008;25:457-507.

5. National Institute for Health and Care Excellence. Interventional procedure guidance [IPG472] 2012. http://www.nice.org.uk/ guidance/ipg472.

6. Kernan WN, Ovbiagele B, Black HR, et al. Guidelines for the prevention of stroke in patients with stroke and transient ischemic attack: a guideline for healthcare professionals from the American Heart Association/American Stroke Association. Stroke 2014;45:2160-236.

7. Lew KN, Angelini GD, Hollingworth W. A time-series study of percutaneous closure of patent foramen ovale: premature adoption? Open Heart 2016;3:e000313.

8. Meier B, Kalesan B, Mattle HP, et al. Percutaneous Closure of Patent Foramen Ovale in Cryptogenic Embolism. N Engl J Med Overseas Ed 2013;368:1083-91.

9. Carroll JD, Saver JL, Thaler DE, et al. Closure of Patent Foramen Ovale versus Medical Therapy after Cryptogenic Stroke. N Engl J Med Overseas Ed 2013;368:1092-100.

10. Rengifo-Moreno P, Palacios IF, Junpaparp P, et al. Patent foramen ovale transcatheter closure vs. medical therapy on recurrent vascular events: a systematic review and meta-analysis of randomized controlled trials. Eur Heart J 2013;34:3342-52.

11. Thaler DT. RESPECT: Final long-term outcomes from a prospective, randomized trial of PFO closure in patients with cryptogenic stroke. Washington, DC, 2016. TCT 2016; November 1, 2016.

12. Kent DM, Thaler DE. RoPE Study Investigators. The Risk of Paradoxical Embolism (RoPE) Study: developing risk models for application to ongoing randomized trials of percutaneous patent foramen ovale closure for cryptogenic stroke. Trials 2011;12:185. 